\title{
Low frequency noise in resonant Josephson soliton oscillators
}

\author{
Hansen, Jørn Bindslev; Holst, T.; Wellstood, Frederick C.; Clarke, John
}

Published in:

I E E E Transactions on Magnetics

Link to article, DOI:

$10.1109 / 20.133928$

Publication date:

1991

Document Version

Publisher's PDF, also known as Version of record

Link back to DTU Orbit

Citation (APA):

Hansen, J. B., Holst, T., Wellstood, F. C., \& Clarke, J. (1991). Low frequency noise in resonant Josephson soliton oscillators. I E E E Transactions on Magnetics, 27(2, 4), 3343-3346. https://doi.org/10.1109/20.133928

\section{General rights}

Copyright and moral rights for the publications made accessible in the public portal are retained by the authors and/or other copyright owners and it is a condition of accessing publications that users recognise and abide by the legal requirements associated with these rights.

- Users may download and print one copy of any publication from the public portal for the purpose of private study or research.

- You may not further distribute the material or use it for any profit-making activity or commercial gain

- You may freely distribute the URL identifying the publication in the public portal

If you believe that this document breaches copyright please contact us providing details, and we will remove access to the work immediately and investigate your claim. 


\title{
LOW FREQUENCY NOISE IN RESONANT JOSEPHSON SOLITON OSCILLATORS.
}

\author{
J. Bindslev Hansen*, T. Holst* ${ }^{*}$ Frederick C. Wellstood and John Clarke \\ Department of Physics, University of California \\ and \\ Materials and Chemical Sciences Division, \\ Lawrence Berkeley Laboratory, \\ Berkeley, California, 94720 USA \\ * Physics Laboratory I / MIDIT \\ The Technical University of Denmark \\ DK-2800 Lyngby, Denmark
}

\begin{abstract}
The noise in the resonant soliton mode of long and narrow Josephson tunnel junctions (Josephson Transmission Lines, JTLs) has been measured in the frequency range from $0.1 \mathrm{~Hz}$ to $25 \mathrm{kHz}$ by means of a dc SQUID. To within a factor of 2 the measured white noise was found to be equal to the Nyquist voltage noise in a resistance equal to the dynamic resistance $R_{D}$ of the current-voltage characteristic at the bias point. In contrast, measurements of the linewidth of the microwave radiation from the same JTL showed that the spectral density of the underlying noise voltage scaled as $R_{D}{ }^{2} / R_{S}$ where $R_{S}$ is the static resistance. The origin of the different behavior is not known.
\end{abstract}

\section{Introduction}

Long, one-dimensional Josephson tunnel junctions (Josephson Transmission Lines, JTL's) are of considerable theoretical and experimental interest because of their highly nonlinear characteristics 1,2 . These devices are slow-wave $(\bar{c} \approx$ $0.025 \mathrm{c}$ ), nonlinear transmission lines that support a variety of excitations, such as fluxons, plasmons and breathers, and are modelled by the perturbed, thermal sine-Gordon equation 3,4 . Soliton (fluxon) devices may find applications in radiofrequency oscillators and amplifiers and in digital circuits where the soliton represents one bit of information 5 . The low frequency noise is of interest because it determines the performance of the amplifier and the linewidth of the soliton oscillator. Few noise studies have been reported ${ }^{6}$, however, and here we describe a series of measurements using a dc Superconducting Quantum Interference Device (SQUID).

\section{JTL model}

On a JTL, the soliton is a localized $2 \pi$ "kink" in the phase difference $\delta(x, t)$ between the two superconducting electrodes on either side of the tunnel barrier. The phase kink corresponds to one quantum of flux, $\Phi_{O}=h / 2 e$, (one fluxon or one antifluxon) threading the junction in the plane of the barrier. Resonant motion of these fluxons or antifluxons produces current singularities in the current-voltage (I-V) characteristics, namely zero-field steps (ZFSs) at voltages ${ }^{7} V_{n}$

Manuscript received September 24, 1990

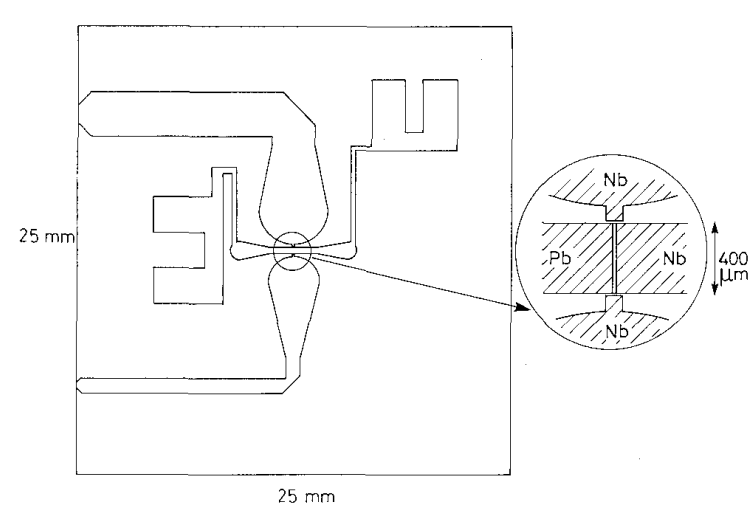

Figure 1. Configuration of $\mathrm{Nb}-\mathrm{Nb} \mathrm{O}_{\mathrm{X}}-\mathrm{Pb}$ overlap JTL showing coupling to microstrip antennas.

$=\mathrm{n} \Phi_{0} \bar{c} / L ;$ here $\mathrm{n}=1,2,3, \ldots, \mathrm{L} / \lambda_{\mathrm{J}}$, where $\mathrm{L}$ is the junction length and $\lambda_{\mathrm{J}}$ is the Josephson penetration depth. On the $\mathrm{n}=$ 1 step the fundamental frequency of the pulse train emitted from one end of the JTL is $f_{1}=V_{1} / 2 \Phi_{O}=\bar{c} / 2 L$ with a linewidth $\Delta \mathrm{f}_{1}$ determined by the fluctuations in the fluxon velocity. Assuming a Nyquist noise spectrum for the junction, one finds 4 the full linewidth at half power for a current-biased JTL to be

$$
\Delta f_{1}=\pi k_{B} T R_{D}^{2} / R_{S} \Phi_{o}^{2}
$$

where $R_{D}$ is the dynamic resistance and $R_{S}=V / I$, and $T$ is the temperature. The form of Eq. (1) can be understood as follows. The Nyquist noise current associated with the static resistance has spectral density $4 \mathrm{k}_{\mathrm{B}} \mathrm{T} / \mathrm{R}_{\mathrm{S}}$, and is converted to a voltage spectral density by the factor $\mathrm{RD}^{2}$. The fluctuations in voltage then produce fluctuations in the Josephson frequency.

We are not aware of any calculations of the low frequency white noise. One might expect both mixed-down noise 8 and pair fluctuations arising from photon number fluctuations ${ }^{9}$ to give additional contributions to the noise generated at the measurement frequency. Given the lack of a theory for this complicated, nonlinear system, we shall find it convenient to analyze our results in terms of an effective noise resistance $R^{*}$, so that $S_{V}(f)=4 k_{B} T R^{*}$. 


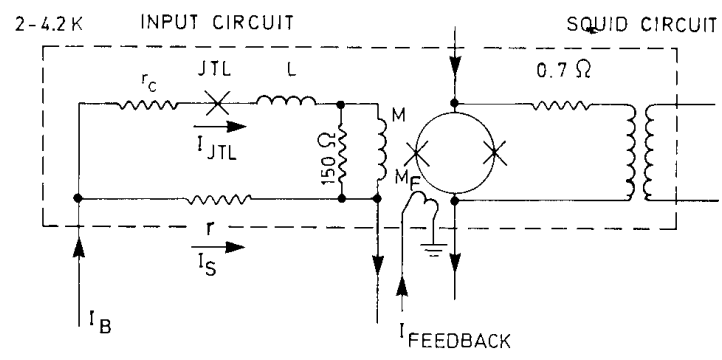

Figure 2. Schematic of dc SQUID amplifier circuit used to measure low frequency voltage noise. Typical circuit parameters were $\mathrm{r}=0.030 \Omega, \mathrm{r}_{\mathrm{C}}=10-40 \mathrm{~m} \Omega$, $\mathrm{L}=9.6 \mu \mathrm{H}, \mathrm{M}=15.5 \mathrm{nH}$ and $\mathrm{MF}_{\mathrm{F}}=37 \mathrm{pH}$.

\section{Experimental details}

The JTLs, coupled to microstrip antennas (Fig. 1), were fabricated on Corning 7059 glass at TUD. The $\mathrm{Nb}-\mathrm{NbO} \mathrm{O}_{\mathrm{X}} \mathrm{Pb}$ overlap junctions were $400 \times 20 \mu \mathrm{m}^{2}$ with critical current densities in the range 10 to $40 \mathrm{~A} / \mathrm{cm}^{2}$; the corresponding values of $\lambda_{\mathbf{J}}$ were $145-75 \mu \mathrm{m}$. Four samples were used in this study.

The $\mathrm{dc}$ and microwave characteristics were determined at TUD in a carefully screened cryostat with heavily filtered leads. When we changed the bias point from the bottom to the top of a given ZFS, we found the linewidth of the soliton radiation at about $10 \mathrm{GHz}$ varied from about $1 \mathrm{MHz}$ to 2 $\mathrm{kHz}$, always a factor of 2-3 larger than the theoretical prediction obtained with measured values of $T, R_{D}$ and $R_{S}$.

The low frequency noise was measured at UCB using the dc SQUID amplifier shown in Fig. 2. The 50-turn input coil of the planar SQUID 10,11 was connected in series with the junction and a resistor $r=0.026 \Omega ; r_{c}(10-40 \mathrm{~m} \Omega)$ is the contact resistance of the In pads on the junction. At the low frequencies, $\mathrm{f}$, at which the measurements were made $(\mathrm{f}<25$ $\mathrm{kHz}$ ) the JTL was voltage biased for $\mathrm{R}_{\mathrm{D}}>>\mathrm{r}$ and current biased for $r>R_{D}$; at the soliton frequencies the JTL was most likely current biased. The SQUID was operated in a conventional flux-locked loop in which the output voltage was proportional to the current in the input coil. Both the SQUID and the JTL were mounted in a $\mathrm{Nb}$ cell filled with and immersed in liquid helium, and the cryostat was surrounded by two $\mu$-metal shields. The leads were heavily filtered and all circuits were battery powered; a shielded room enclosed the entire experiment.

\section{Experimental results}

The I-V characteristics of each JTL were obtained from plots of the SQUID output vs. the bias current IB in the input circuit. A typical I-V characteristic reconstructed from these data is shown in Fig. 3. The noise was measured over the range $0.1 \mathrm{~Hz}$ to $25 \mathrm{kHz}$, and five representative spectra measured at points indicated in Fig. 3 are shown in Fig. 4. Spectrum (a), obtained with zero bias in the JTL, represents

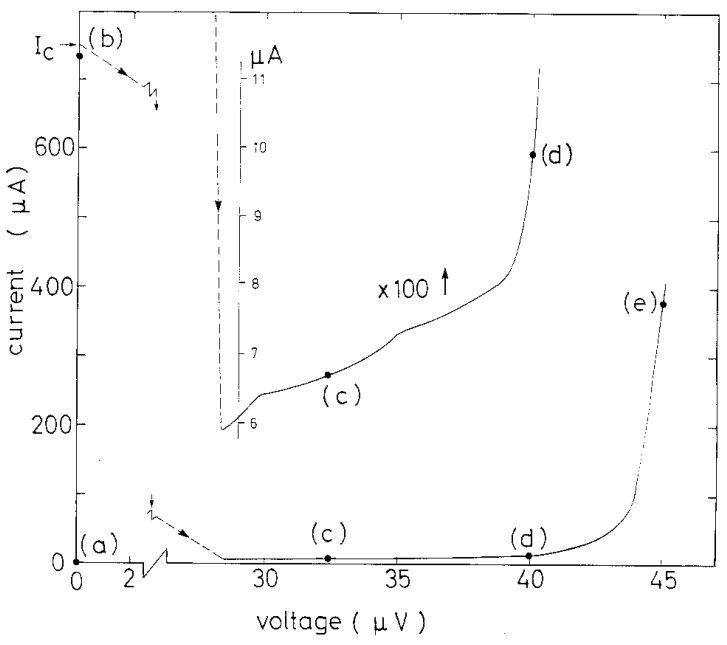

Figure 3. Current-voltage characteristic of JTL at $\mathrm{T}=4.2 \mathrm{~K}$ showing a ZFS at about $43 \mu \mathrm{V}$. Letters (a)-(e) denote bias points at which the noise spectra in Fig. 4 were obtained. Dashed lines are load-lines resulting from the circuit resistance loading the JTL.

the Nyquist noise from the resistance $r+r_{c}$. The roll-off at about $2 \mathrm{kHz}$ corresponds to the low-pass filter knee of the input circuit. Spectrum (b) was obtained with a current of 730 $\mu \mathrm{A}$ in the JTL, which was still in the zero voltage state. The additional noise below about $5 \mathrm{~Hz}$ arises from drift in the bias current. In the zero voltage state, we verified that the white noise power scaled with the temperature with a magnitude predicted by the Nyquist formula to within $\pm 5 \%$. This result implies that extraneous noise was negligible.

Spectrum (c) was obtained with the JTL biased at about $6.7 \mathrm{HA}$ on the quasiparticle tunneling curve; note that the level of white noise was appreciably lower than in spectra (a) and (b) because the total current resistance increased from ( $\mathrm{r}$ $\left.+r_{C}\right)=0.038 \Omega$ to about $5 \Omega$. In spectrum (d) we observe a marked peak in the noise at a few kilohertz. This peak arose from random telegraph signals (RTSs) across the JTL with typical magnitude of 10-20 nV. These RTSs are associated with unstable bias points where apparently two different soliton modes compete. Finally, spectrum (e) shows the noise near the top of the first $(n=1)$ ZFS, where, typically, $R_{S}=0.1$ $\Omega$ and $\mathrm{R}_{\mathrm{D}}=2 \mathrm{~m} \Omega$. The noise is white at low frequencies $(\mathrm{f}<10 \mathrm{~Hz}$ ) and between roughly 100 and $700 \mathrm{~Hz}$.

To characterize the noise, we write the spectral density of the noise current in the input circuit as:

$$
S_{l}(f)=4 k_{B} T\left(R^{*}+r+r_{c}\right) /\left(R_{D}+r+r_{c}\right)^{2}
$$

Here, we have assumed that the voltage noise source of the JTL can be characterized by the effective resistance $R^{*}$, and that the total voltage noise power should be divided by the dynamic resistance of the input circuit to obtain the total current noise power. We note that for bias points near the top of the ZFSs where $R_{D}$ is a few milliohms the contribution of the junction noise is small, and the error in its 


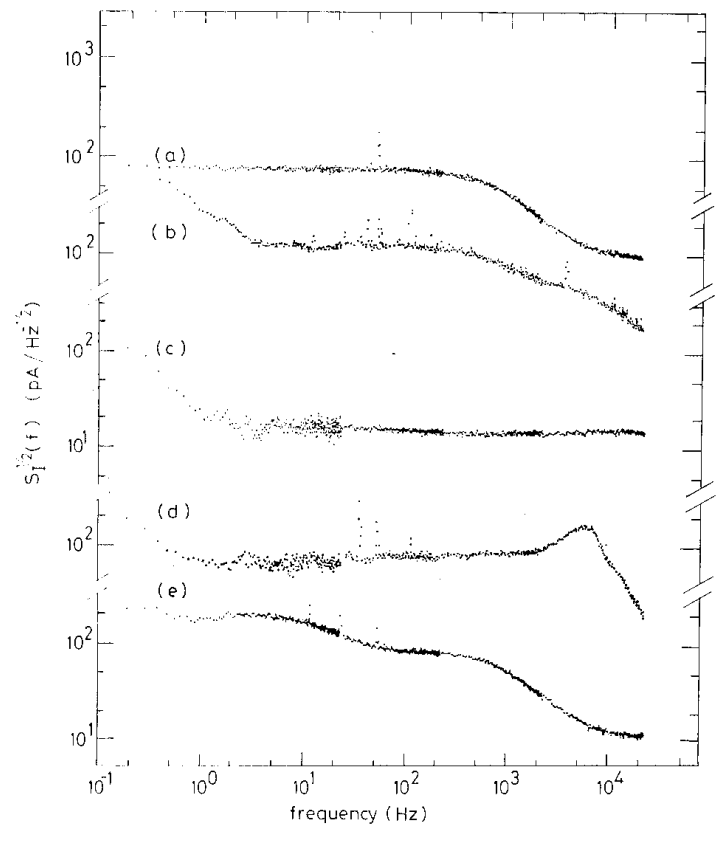

Figure 4. Measured $4.2 \mathrm{~K}$ noise spectra $\mathrm{S}_{\mathbf{I}}{ }^{1 / 2}(\mathrm{f})$ for JTL. Letters (a)-(e) refer to the bias points in Fig. 3.

measurement correspondingly large. The results are shown in Fig. 5 where we plot $\mathrm{R}^{*} / \mathrm{R}_{\mathrm{D}}$, inferred from Eq. (2), vs. current in the JTL. For reference we also plot in Fig. 5 the measured values of $R_{D}, R_{S}, R_{D} / R_{S}$, and the value of $R^{*}$ inferred from the noise measurements and $R_{D}$.

On the quasiparticle curve (filled circles) $R^{*} / R_{D}$ varies from about 2 to 5 . Since these results are in the limit hf $\approx$ $\mathrm{eV}<<\mathrm{k}_{\mathrm{B}} \mathrm{T}$, one would expect the noise power to be given by the Nyquist formula $4 k_{B} T_{D}$. Thus, it is likely that the excess noise is produced by the dynamics of the long junction; we are not aware of any theory for this noise. On the ZFS, on the other hand, $\mathrm{R}^{*} / \mathrm{R}_{\mathrm{D}}$ is between approximately 1 and 2 . Yet, as shown in Fig. 5, over the current range $6-380 \mu \mathrm{A}$ $\mathrm{R}_{\mathrm{D}} / \mathrm{R}_{\mathrm{S}}$ varies from about 1 (low currents) to about 0.02 (high currents). Thus, we conclude that at least at high currents on the ZFS, the effective noise resistance $R^{*}$ is not given by $R_{D}^{2} / R_{S}$ as is the case for the linewidth [Eq.(1)]. Rather, it appears that to a first approximation $R^{*} \approx R_{D}$. From this result, we conclude that the noise at low frequencies is higher than one would expect from a naive interpretation of Eq.(1), that is, the factor $R_{D} / R_{S}$ is absent. Since $R_{D} / R_{S}$ is typically 0.03 on the ZFS1 (Fig. 5), the low frequency noise power would be reduced by about 30 if this factor were present.

\section{Concluding remarks}

We have measured the soliton oscillator linewidth and low frequency noise, of a JTL biased on the first ZFS. At an oscillator frequency of $10 \mathrm{GHz}$, the linewidth is given by $\alpha$ Ir $\mathrm{k}_{\mathrm{B}} \mathrm{TR}_{\mathrm{D}}{ }^{2} / \mathrm{R}_{\mathrm{S}}{ }^{2}{ }_{0}^{2}$ over a current bias range in which $R_{D}{ }^{2} / R_{S}$ varies by about a factor of 500 . The coefficient $\alpha$ is
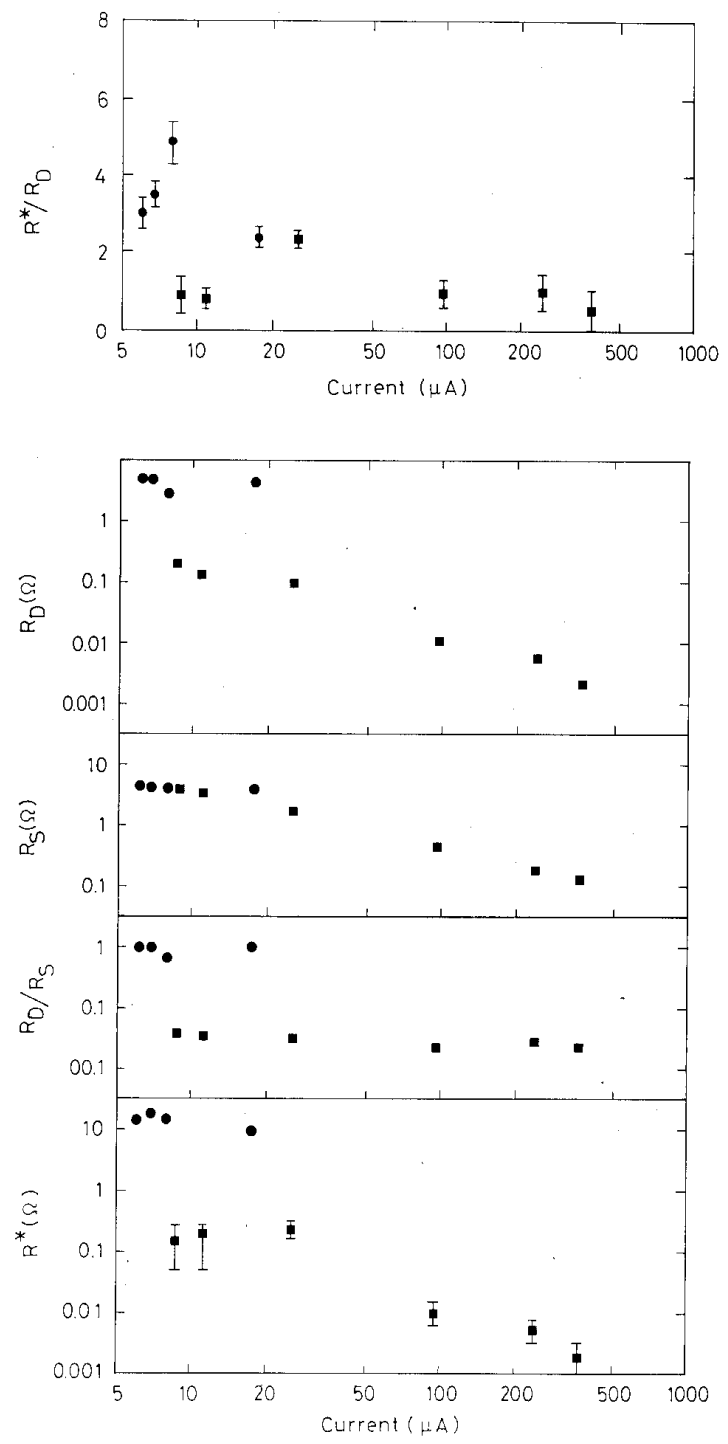

Figure 5. Upper plot shows current-dependence of normalized noise resistance $\mathrm{R}^{*} / \mathrm{R}_{\mathrm{D}}$ calculated from measured data at $4.2 \mathrm{~K}$ using Eq. (2). Filled circles refer to quasiparticle curve; filled squares refer to the first ZFS. Lower plots show current-dependence at $4.2 \mathrm{~K}$ of the measured values of $R_{D}, R_{S}$ and $R_{D} / R_{S}$ and of the inferred $R^{*}$.

typically 2-3; the reason for this excess linewidth is not known. The spectral density of the low frequency white voltage noise is given by approximately $\beta 4 \mathrm{k}_{B} \mathrm{TR}_{\mathrm{D}}$ where $\beta$ is typically $1-2$. Over the range of current studied $\mathrm{R}_{\mathrm{S}}$ varied by about a factor of 50, and thus appears not to enter into the magnitude of the voltage noise. The difference in the dependence of the linewidth and low frequency noise on $\mathrm{R}_{\mathrm{S}}$ is not understood, and it would be of considerable interest to develop a detailed theory for the mechanism of low frequency noise. 


\section{Acknowledgment}

We thank Michael Heaney, Inge Rasmussen and Søren Hjorth for help and advice and Mogens Samuelsen, Henrik Svensmark, Jesper Mygind and Mario Salerno for fruitful discussions. JBH and TH thank the Physics Department, University of California, Berkeley, and Lawrence Berkeley Laboratory for hospitality during the course of the experimental work and The Danish Natural Science Research Council, NATO Science Fellowship Programme, the Otto Mønsted, the Knud Højgaard and the Carlsberg Foundations for grants. This work was supported by the Director, Office of Energy Research, Office of Basic Energy Sciences, Material Sciences Division of the U.S. Department of Energy under Contract No. DE-AC03-76SF00098.

\section{References}

1. N.F. Pedersen, "Josephson fluxon devices", presented at this conference.

2. A. Matsuda and T. Kawakami, "Fluxon propagation on a Josephson transmission line", Phys. Rev. Lett. 51, 694 , 1983.

3. D.W. MacLaughlin and A.C. Scott, "Perturbation analysis of fluxon dynamics", Phys. Rev. A 18, 1652, 1978.

4. E. Joergensen, V.P. Koshelets, R. Monaco, J. Mygind, M.R. Samuelsen and M. Salerno, "Thermal fluctuations in resonant motion of fluxons on a Josephson transmission line", Phys. Rev. Lett. 49, 1093, 1982.

5. K.K. Likharev, V.K Semenov and A.B. Zorin, "New possibilities for superconducting devices", in Modern Superconducting Devices, S.T. Ruggiero and D.A. Rudman, Eds., Academic Press, Boston, 1990.

6. B.S. Han, B. Lee, O.G. Symko, W.J. Yeh, and D.J. Zheng, "Noise characteristics and instabilities of long Josephson junctions", IEEE Trans. Magn. MAG-25, 1396, 1989.

7. T.A. Fulton and R.D. Dynes, "Single vortex propagation in Josephson tunnel junctions", Sol. State Comm. 12, 57, 1973.

8. K.K. Likharev and V.K. Semenov, "Fluctuation spectrum in superconducting point contact", Zh. Eksp. Teor. Fiz. Pis'ma Red. 15, 625, 1972 (JETP Lett. 15, 442, 1972); R.H. Koch, D.J. Van Harlingen and J. Clarke, "Measurements of quantum noise in resistively shunted Josephson junctions", Phys. Rev. B 26, 74, 1982.

9. M.J. Stephen, "Noise in the ac Josephson effect", Phys. Rev. 182, 531, 1969.

10. J.M. Martinis and J. Clarke, "Measurements of current noise in dc SQUIDs", IEEE Trans. Magn. MAG-19, 446, 1983; C. Hilbert and J. Clarke, "Radio-frequency amplifier based on a dc SQUID", Appl. Phys. Lett. 40, $736,1983$.

11. F. Wellstood, C. Heiden and J. Clarke, "Integrated dc SQUID magnetometer with a high slew rate", Rev. Sci. Instrum. 55, 952, 1984; F.C. Wellstood, C. Urbina, and J. Clarke, "Excess noise in dc SQUIDs from $4.2 \mathrm{~K}$ to 0.022 K", IEEE Trans. Magn. MAG-23, 1662, 1987. 